\title{
Effect of the Oral Administration of Fungal Ligands in a Murine Model of DSS-Induced Colitis
}

\author{
Daniel Gozalbo ${ }^{1}$, M. Pilar Falomir ${ }^{1}$, Alberto Yáñez ${ }^{1}$, M. Luisa Gil ${ }^{1}$ and Celia Murciano ${ }^{*}, 2$ \\ ${ }^{I}$ Departamento de Microbiología y Ecología, Universitat de València, C/ Dr. Moliner 50, E-46100 Burjassot, Spain \\ ${ }^{2}$ Department of Oral Immunology, King's College London Dental Institute, King's College London, London, SE1 9RT, \\ United Kingdom
}

\begin{abstract}
Background: The molecular interaction between ligands of commensal microbiota and host receptors, such as Toll-like receptors (TLRs), at the surface of the epithelial cells of the gastrointestinal (GI) tract plays a critical role in its homeostasis and protection. The aim of this work was to determine the usefulness of the oral administration of yeast cells or soluble yeast-derived ligands for TLRs in the protection and modulation of the inflammatory response of the GI tract in a murine model of experimentally induced colitis. Materials and Methods: C57BL/6 mice were given 3\% dextran sulphate sodium salt (DSS) to induce experimental colitis. Protection assays were performed by oral administration of viable Saccharomyces spp. yeasts cells, soluble yeast mannan or PBS (control), starting either after or during the DSS treatment. The in vitro production of cytokines was measured in colon segments of selected mice, either in the presence or absence of heat-inactivated yeasts or mannan. Results: In mice with experimental acute or sublethal colitis, no statistically significant differences were found between yeast/mannan treated mice and controls, both for mortality and loss of weight. The in vitro production of cytokines was not altered by the addition of mannan or yeasts to the culture, in both healthy mice and mice with induced colitis. Conclusions: Direct oral administration of viable yeasts or soluble yeast mannan does not show any protective effect during disease in a model of experimentally induced colitis in immunocompetent mice.
\end{abstract}

Keywords: Cytokines, colitis, DSS, fungal ligands, mannan, Saccharomyces spp.

\section{INTRODUCTION}

Inflammatory bowel disease (IBD) is a chronic inflammatory disorder of the gastrointestinal (GI) tract, being Crohn's disease (CD) and ulcerative colitis (UC) the two major forms of the disease. The exact causes and mechanisms underlying $\mathrm{CD}$ and $\mathrm{UC}$ are not completely understood, although it is now accepted that IBD is a dysregulated immune response that occurs in genetically susceptible individuals as the result of a complex interaction among environmental factors, host microbiota and the intestinal immune system leading to continuous activation of the immune response; therefore most treatments for IBD involve suppressing or altering this aberrant immune response and the role of intestinal microbiota in the pathobiology of IBD is becoming more evident [1-3].

Several animal models have been developed to understand the pathology of IBD. It has been described that mice receiving dextran sulphate sodium salt (DSS) orally developed acute colitis resembling UC. The DSS administration to mice causes the development of colonic mucosal inflammation with ulcerations, body weight loss and bloody diarrhoea [4]. However, the mechanisms by which DSS induces colitis are not fully clear. Some of the proposed mechanisms are toxic effects on the epithelium, increased exposure to

*Address correspondence to this author at the Department of Oral Immunology, Floor 28, Tower Wing, King's College London Dental Institute, King's College London, London, SE1 9RT, United Kingdom; Tel: +442071888707; Fax: +442071884375;

E-mail: celia.murciano@kcl.ac.uk luminal antigens by destruction of mucin content or altered macrophage function due to ingestion of DSS [5].

Recently, focus has been placed on probiotic and prebiotic therapies for the treatment of IBD in order to reduce intestinal inflammation and restore the balance to the gastrointestinal microbiota [2]. Probiotics have been described as "living organisms in food and dietary supplements which, upon ingestion, improve the health of the host beyond their inherent basic nutrition" [6]. There are a large number of probiotic species identified, most of which are lactic acid bacteria, such as Lactobacillus and Bifidobacterium species, but also the fungal species Saccharomyces boulardii. Although most of the species described as probiotics have different action mechanisms, all present beneficial properties, including the ability to improve epithelial barrier function, alter the intestinal microbiota and modulate the mucosal immune system [7].

Toll-like receptors (TLRs) constitute a family of patternrecognition receptors (PPRs) that function as sensors of infection being critical for the initiation of inflammatory and innate immune defense responses and also for the modulation of adaptive immune responses. TLR ligands include molecular products derived from bacteria, protozoa, viruses and fungi $[8,9]$. A novel function was described for TLRs expressed at the surface of epithelial cells of the GI tract as they are involved in the protection from direct epithelial injury and participate in the maintenance of epithelial homeostasis $[10,11]$. Some possible mechanisms, not mutually exclusive, for the TLR-mediated protection are (i) the steady-state induction of protective factors, via the consti- 
tutive detection of lumenally derived TLR ligands on commensals by TLRs expressed on colonic epithelium, and (ii) the production of protective factors in response to commensal-derived TLR ligands upon epithelial damage [10]. Therefore the recognition of commensal bacterial pro-ducts by TLRs has a beneficial and crucial role in mammalian physiology. Further evidence has confirmed the protective effect on DSS-induced colitis of the TLR-mediated signalling in mouse models [12-14]; Myd88 (and adaptor molecule that mediates signalling through TLRs) in nonhematopoietic cells protects mice against induced colitis by regulating specific epithelial growth factor receptor ligands [15], and regulation of colonic epithelial repair in mice involves TLR4 and endogenous hyaluronic acid expression [16,17].

It is well established that yeast cells of the opportunistic fungal pathogen species Candida albicans contain at their cell wall microbial-associated molecular patterns that are recognized by TLRs (mainly TLR2 and TLR4) that mediate innate immune responses such as inflammation [18,19], and that the cell wall composition and organization are similar both in C. albicans and $S$. cerevisiae yeasts cells [20]. Therefore, yeast cells or soluble yeast-derived ligands for TLRs may also be involved in modulating the inflammatory response of the GI tract, and as a consequence may have a potential therapeutic effect during IBD. In this context, controlled clinical trials have shown that the probiotic species $S$. boulardii is effective in the prevention and/or treatment of various intestinal disorders including recurrent Clostridium difficile disease, acute diarrhea in adults and children, antibiotic-associated diarrhoea and relapses of CD or UC $[21,22]$, and also, in a mouse model of chemicallyinduced colitis, $S$. boulardii has been shown to decrease inflammation and intestinal colonization by C. albicans [23]. The relationship between yeast antigens and gastrointestinal inflammatory disorders is further supported since antibodies directed against particular oligomannose sequences present in yeast mannan, termed anti-S. cerevisiae antibodies (ASCAs) are markers of CD but not of UC, and that $C$. albicans is an immunogen for ASCA markers of CD [24,25].

In this work, using a murine model of experimentally DSS-induced colitis, we have studied the effect of the oral administration of $S$. cerevisiae, $S$. boulardii and soluble yeast cell wall-derived mannan in the protection from injury of GI tract during inflammatory disease.

\section{MATERIALS AND METHODOLOGY}

\section{Mice}

Female C57BL/6 mice (8-10 weeks old) were purchased from Harlan Ibérica (Barcelona, Spain), and were bred and maintained under specific pathogen-free conditions at the animal facility of the University of Valencia during the experimental assays. All assays involving mice were approved by the Institutional Animal Care and Use Committee.

\section{Induction of DSS Colitis}

To induce experimental acute colitis, mice were given 3\% Dextran Sulphate Sodium Salt (DSS) (36,000 - 50,000 $\mathrm{kDa}$; MP Biomedicals Europe, Illkirch, France), ad libitum, in their drinking water for seven days and then switched to regular drinking water. To induce sublethal colitis, mice were given $3 \% \mathrm{DSS}$, ad libitum, in drinking water until a percentage of loss weight between $7 \%$ and $9 \%$, and then switched to regular drinking water. The amount of DSS water consumed per animal was recorded and no differences were observed with the amount of regular drinking water drank by non-treated mice.

For survival studies, mice were followed daily for 17 days and weighed every day for the determination of percent weight change. This was calculated as: $\%$ weight change $=$ [(day X weight - day 0 weight) / day 0 weight $]$ x 100.

Animals were monitored daily, and those with severe rectal bleeding, severe diarrhea or serious general symptoms of morbidity were humanely sacrificed and not included in the experiments.

\section{Yeasts Strains and Culture Conditions}

Two yeasts strains were used in this study: one strain of $S$. cerevisiae var boulardii isolated from a commercial probiotic product [26], and the natural wine yeast strain $S$. cerevisiae CECT (Spanish Type Culture Collection) 10431. These yeast strains were selected as $S$. boulardii is recognized to have biotherapeutic effects on intestinal mucosa, and the wine yeast strain does not express putative virulence traits $[21,26]$.

For protection assays viable cells of $S$. cerevisiae 10431 and $S$. boulardii were grown in YPD medium (1\% yeast extract, $2 \%$ peptone, $2 \%$ glucose) at $28^{\circ} \mathrm{C}$ up to exponential growth phase $\left(A_{600} \mathrm{~nm} 0.4-0.6\right)$ and then collected and washed twice with pyrogen-free phosphate-buffered saline (PBS; Gibco, Barcelona, Spain). Cells were then diluted in PBS to the appropriate cell concentration and kept at $4{ }^{\circ} \mathrm{C}$ before oral administration. Fresh yeasts cultures were prepared every 48 hours.

Mice were orally administered daily with $150 \times 10^{6}$ viable yeasts cells in $0.05 \mathrm{ml}$ PBS or with $5 \mathrm{mg}$ in $0.05 \mathrm{ml}$ PBS of soluble yeast mannan from $S$. cerevisiae (SigmaAldrich, Madrid, Spain).

For the in vitro production of cytokines assays, yeasts cells of $S$. cerevisiae and $S$. boulardii were prepared as described above and then they were heat inactivated. Heatkilling was performed by incubating the cells $\left(20 \times 10^{6}\right.$ cells $/ \mathrm{ml}$ in PBS) at $90^{\circ} \mathrm{C}$ for $1 \mathrm{~h}$, as reported elsewhere [27, 28]. After treatments, cells were washed twice with PBS and inactivation of cells was checked by the absence of growth following incubation of samples $\left(2 \times 10^{6}\right.$ cells $)$ on Sabouraud dextrose agar plates for $48 \mathrm{~h}$ at $28^{\circ} \mathrm{C}$.

\section{Measurement of in vitro Colonic Cytokine Production}

Segments of $0.5 \mathrm{~cm}$ length of proximal and distal colon from selected mice were obtained and were extensively washed with PBS supplemented with $1 \%$ penicillin-streptomycin stock solution (Gibco). The segments were incubated for $24 \mathrm{~h}$ at $37^{\circ} \mathrm{C}$ in a $5 \% \mathrm{CO}_{2}$ atmosphere in $0.5 \mathrm{ml}$ cell culture medium (RPMI 1640 medium supplemented with 1\% penicillin-streptomycin stock solution; Gibco), either in the presence or absence of heat-inactivated $S$. cerevisiae yeasts $\left(50 \times 10^{6}\right)$, heat-inactivated $S$. boulardii yeasts $\left(50 \times 10^{6}\right)$ or yeast mannan $(1.5 \mathrm{mg})$. 
Culture supernatants were then harvested and tested for the production of cytokines [interleukin-6 (IL-6), IL-10 and tumor necrosis factor $\alpha$ (TNF- $\alpha)$ ] using commercial ELISA kits for murine cytokines (eBioscience; San Diego, CA, USA).

\section{Statistical Analysis}

Survival curves were analyzed using the Kaplan-Meyer $\log$ rank test. Student's two-tailed t test was used to compare the percent of weight change and cytokine production.

\section{RESULTS}

\section{Administration of Viable Yeasts does not Protect from Mortality in Mice with Acute Colitis}

We performed survival studies to determine the effect of the oral administration of viable yeasts in the protection from mortality in mice with acute experimental colitis. C57BL/6 mice were treated with 3\% DSS in drinking water for 7 days to induce acute colitis. After the treatment the mice were changed to normal drinking water and for 7 days were orally daily administered with $150 \times 10^{6}$ viable $S$. cerevisiae yeasts, $150 \times 10^{6}$ viable $S$. boulardii yeasts or with PBS as a control. After the DSS treatment the mice showed a weight loss ranging from $15 \%$ to $20 \%$, indicating the development of a severe colitis. As shown in Fig. (1) the mortality of mice treated with either S. cerevisiae yeasts or S. boulardii yeasts was not significantly different when compared with PBStreated mice. By day ten after the start of the treatments $90 \%$ and $87 \%$ of the $S$. cerevisiae and $S$. boulardii treated-mice were died, similarly to the mortality showed by PBS-treated mice ( $83 \%$ by day ten) (Fig. 1).

\section{Oral Administration of Viable $S$. boulardii Yeasts has no Effect on the Mortality and Morbidity of Mice with Sublethal Colitis}

As the treatment of mice with $3 \%$ DSS during 7 days (acute colitis) causes a high loss of weight (15-20\%) and severe colitis symptoms, we performed protection studies in mice with sublethal colitis. To induce a sublethal colitis mice were given 3\% DSS in drinking water until they reached a loss weight between 7\% and $9 \%$. Mice were then changed to normal drinking water and were daily orally administered with $150 \times 10^{6}$ viable $S$. boulardii yeasts or with PBS, as a control, for 10 days. In order to reduce the number of animals used, and as we had not found differences in mortality between the oral administration of $S$. cerevisiae and $S$. boulardii yeasts in mice with acute colitis, we performed protections assays using only the $S$. boulardii strain. We choose $S$. boulardii because it has been previously described as a biotherapeutic agent in the prevention and treatment of antibiotic-associated diarrhoea and colitis in humans [22].

Results showed no significant differences, both in mortality and in the percentage of loss weight, between the PBS and the S. boulardii-administered mice (Fig. 2). Mice with sublethal colitis showed a higher survival rate as compared with mice with acute colitis (Fig. 1) $(\sim 81 \%$ and $\sim 14 \%$, respectively), which confirmed a lower degree of intestinal tissue damage. The survival rate of PBS-administered mice was $93 \%$ at day 10 after the start of the treatment, whereas the $S$. boulardii-administered mice showed a survival of $70 \%$ at day 10 (Fig. 2A). This minor difference was not statistically significant. No significant differences in the percentage of weight change during the treatments were observed (Fig. 2B). Both groups of animals started the treatments with an average weight loss of $7.5 \%$, and during the first 6 days of treatment they continued losing weight until a maximum of $24 \%$ in the PBS-treated mice and $28 \%$ in the $S$. boulardii-treated mice. From day 7 until the end of the treatment, surviving mice progressively increased their weight, indicating the recovery from colitis in both groups of mice.

The similar survival rates and kinetics in the percentage of weight change between the S. boulardii-treated and control mice indicate that the oral administration of viable

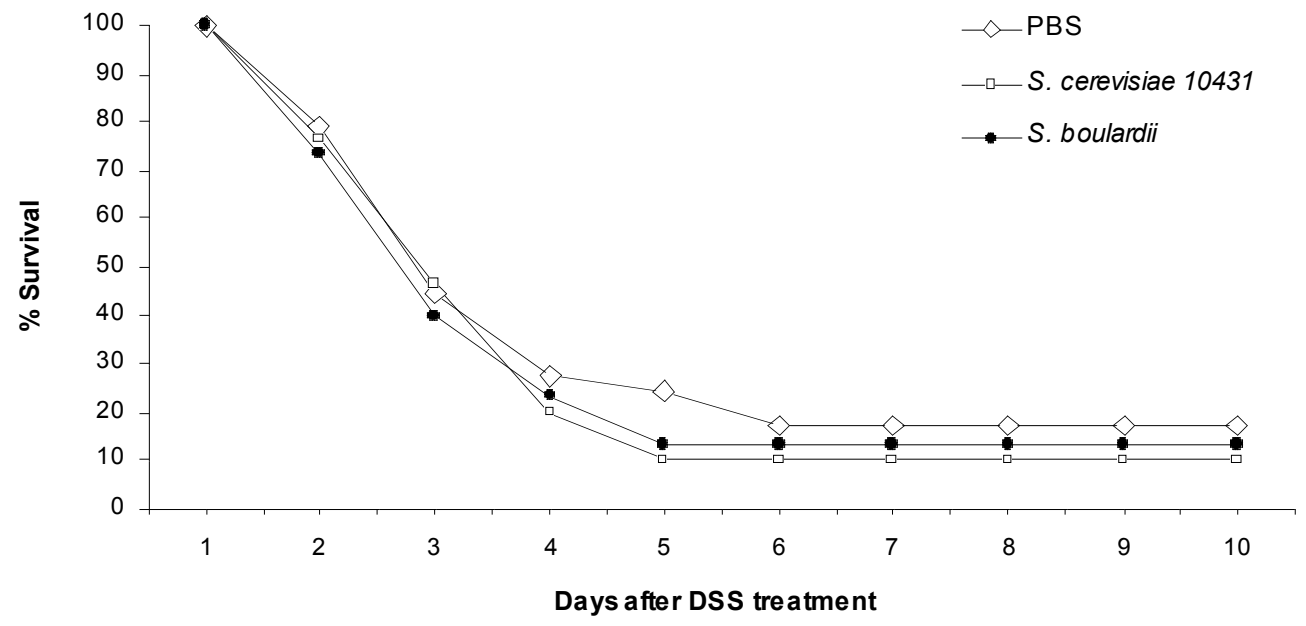

Fig. (1). Survival of mice with experimental acute colitis and treated with $\boldsymbol{S}$. cerevisiae or $\boldsymbol{S}$. boulardii. C57BL/6 mice were given $3 \%$ DSS in drinking water for seven days to induce acute experimental colitis. At day 0 mice were changed to normal drinking water and were daily orally administered with $150 \times 10^{6} \mathrm{~S}$. cerevisiae 10431 viable yeast cells in $0.05 \mathrm{ml} \mathrm{PBS}(\mathrm{n}=30), 150 \times 10^{6} \mathrm{~S}$. boulardii viable yeast cells in $0.05 \mathrm{ml}$ PBS $(\mathrm{n}=30)$ or $0.05 \mathrm{ml}$ PBS (control) $(\mathrm{n}=29)$ during 7 days. Survival was monitored up to day 17 after the start of the DSS treatment. Data are representative of three independent experiments. 

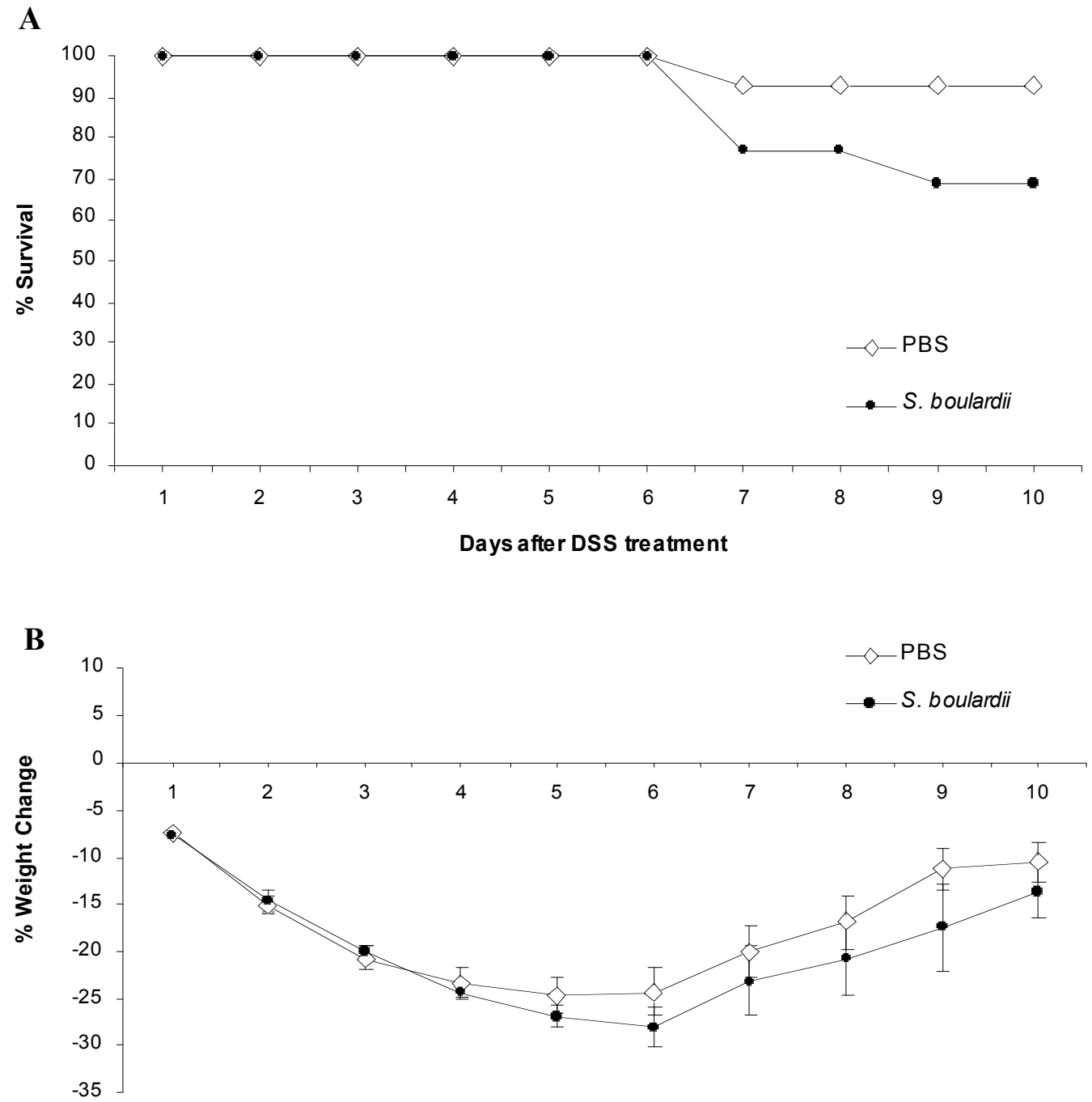

Days after DSS treatment

Fig. (2). Mortality and morbidity of mice with experimental sublethal colitis and treated with $\boldsymbol{S}$. boulardii. C57BL/6 mice were given $3 \%$ DSS in drinking water until a percentage of weight loss between $7 \%$ and $9 \%$. At day 0 mice were changed to normal drinking water and were daily orally administered with $150 \times 10^{6} \mathrm{~S}$. boulardii viable yeast cells in $0.05 \mathrm{ml}$ PBS ( $\mathrm{n}=14$ ) or $0.05 \mathrm{ml}$ PBS (control) (n=14) during 7 days. Survival was monitored up to day 17 after the start of the DSS treatment (A). Surviving mice were weighed daily and the percentage of weight change of each mouse with respect to their weight on the starting day of the DSS treatment was calculated (B). Data are representative of three independent experiments (+/- SEM).

yeasts has no effect in the amelioration of the disease or its symptoms.

\section{Simultaneous Treatment with DSS and Fungal Ligands has no Effect on the Mortality and Morbidity of Mice with Sublethal Colitis}

In the assays described above we started the oral administration of viable yeasts when the mice had developed a DSS-induced colitis (acute or sublethal), so we hypothesised that one of the reasons the yeasts showed no protective effect could be that they were unable to colonise a GI tract with high level of tissue damage. To address this hypothesis we performed protection assays in which we started the oral administration of viable $S$. boulardii yeasts simultaneously with the start of the DSS treatment. Mice were treated with $3 \%$ DSS during 7 days and then changed to normal drinking water. From the start of the DSS treatment and during the following 13 days mice were daily orally administered with PBS (control), $150 \times 10^{6}$ viable $S$. boulardii yeasts or $5 \mathrm{mg}$ of yeast mannan, a soluble cell wall-derived yeast ligand.

As shown in Fig. (3A), the mortality of mice was again very high due to the development of an acute colitis. Moreover, we did not find any significant differences in the mortality between the 3 groups; the mortality of the PBSadministered mice at day 17 was $96 \%$, whereas the mortality of the $S$. boulardii and mannan-administered mice was $83 \%$ and $86 \%$ respectively.

Furthermore the kinetics of weight change was very similar between the surviving mice from the 3 groups (Fig. 3B); almost the $80 \%$ of mice had died at day 13 , but the 


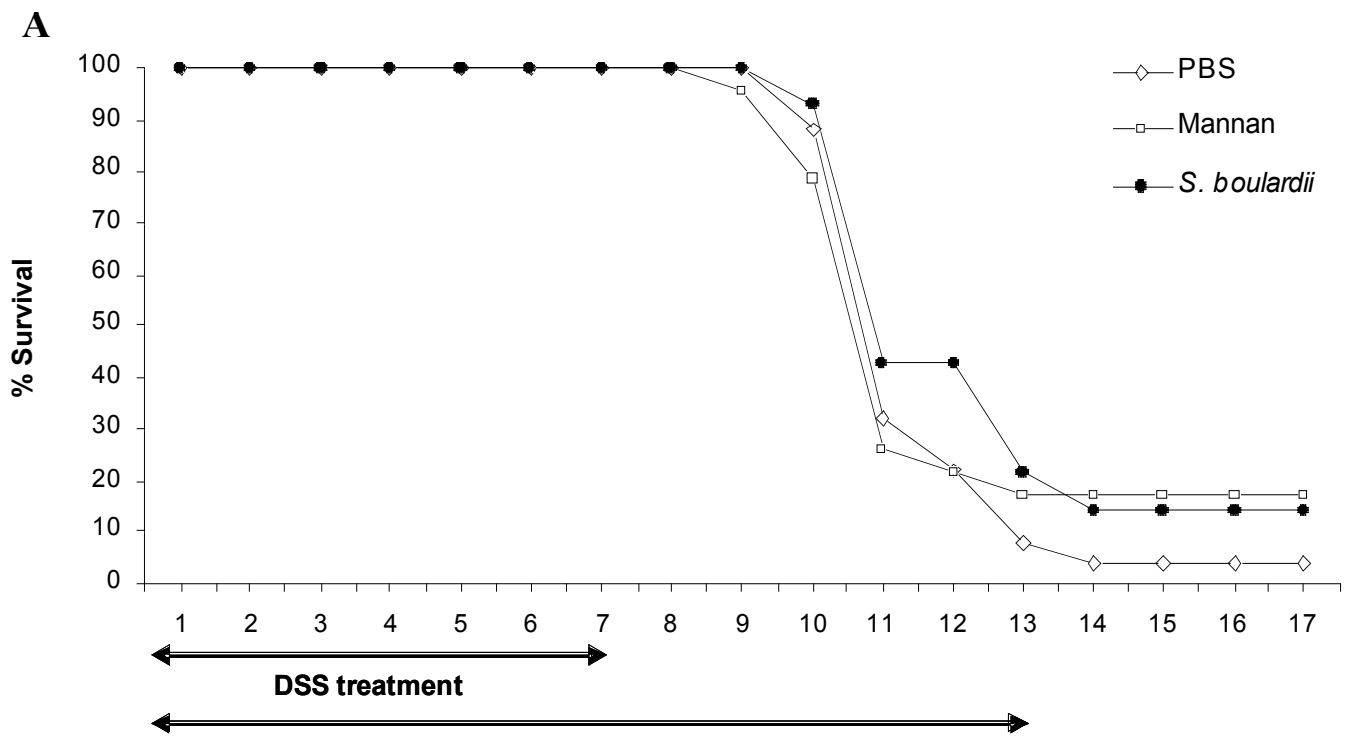

PBS / Mannan / Yeasts administration

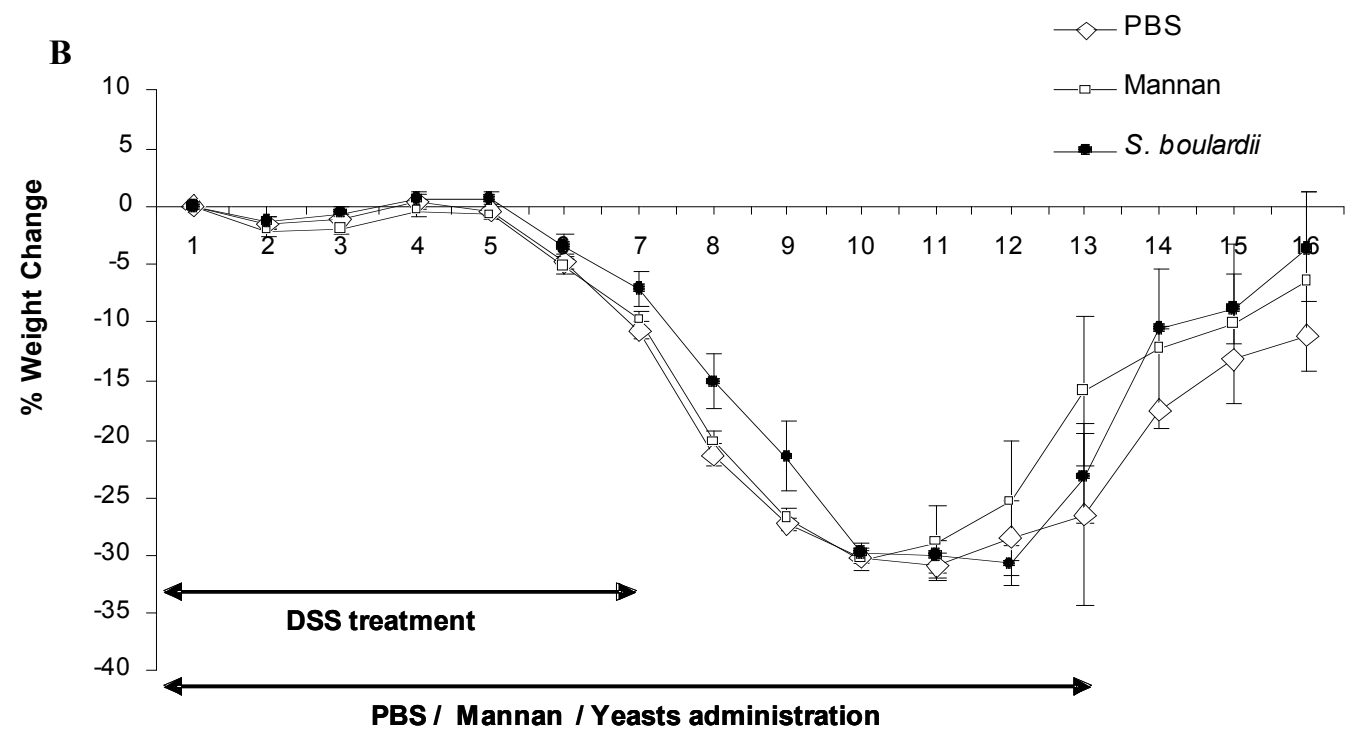

Fig. (3). Mortality and morbidity of mice with experimental acute colitis and simultaneously treated with mannan and $S$. boulardii. C57BL/6 mice were given 3\% DSS in drinking water for seven days to induce acute experimental colitis, and were then changed to normal drinking water. Simultaneously to the DSS treatment mice were daily orally administered with $5 \mathrm{mg}$ of yeast mannan in $0.05 \mathrm{ml}$ PBS ( $\mathrm{n}=23$ ), $150 \times 10^{6} \mathrm{~S}$. boulardii viable yeast cells in $0.05 \mathrm{ml}$ PBS $(\mathrm{n}=14)$ or $0.05 \mathrm{ml}$ PBS (control) (n=25) for 13 days. Survival was monitored up to day 17 after the start of the DSS treatment (A). Surviving mice were weighed daily and the percentage of weight change of each mice with respect to their weight on the day 0 was calculated $(\mathbf{B})$. Data are representative of two independent experiments $(+/-$ SEM).

surviving mice showed a weight recovery similar between the 3 groups.

These results indicate that $S$. boulardii yeasts or the soluble yeast ligand mannan have no in vivo protective effect during the development of an acute colitis.

\section{In Vitro Colonic Production of Cytokines by Mice with Acute Colitis is not Altered in the Presence of Yeasts}

To address the in vitro effect of yeasts in the modulation of the inflammatory response during colitis, we obtained proximal and distal colon segments from mice with acute colitis (treated during 7 days with 3\% DSS). After extensively washes, we cultured the segments with medium alone or in the presence of heat-inactivated $S$. cerevisiae yeasts during $24 \mathrm{~h}$ to further assess the production of the cytokines IL-6, IL-10 and TNF- $\alpha$.

The in vitro production of the proinflammatory cytokine IL-6 was very high both in proximal and distal colon segments [1000 and $2500 \mathrm{pg} \mathrm{ml}^{-1}$ (mg tissue) $)^{-1}$, respectively], indicating a high degree of inflammation in the GI tract after the development of the DSS-induced colitis (Fig. 4). The addition of heat-inactivated $S$. cerevisiae yeasts did not produce any significant effect on IL-6 production (Fig. 4). Compared with IL-6, lower levels of the proinflammatory cytokine TNF- $\alpha$ [up to $8 \mathrm{pg} \mathrm{ml}^{-1}$ (mg tissue) $)^{-1}$ ] and of the anti-inflammatory cytokine IL-10 [up to $100 \mathrm{pg} \mathrm{ml}^{-1}$ (mg 
tissue $)^{-1}$ ] were produced by colonic proximal and distal segments. Again, the addition of heat-inactivated yeasts did not produce any statistical significant effect on the production of cytokines, although a slight decrease was observed in the production of IL-10 and TNF- $\alpha$ by distal colon segments (Fig. 4). Cytokine levels were higher in distal segments compared with proximal segments in all cases, indicating that distal segments show increased injury during experimental colitis, relative to proximal segments.

\section{Yeasts and Mannan do Not Alter the in Vitro Colonic Production of Cytokines}

As the high degree of tissue damage on colon segments from mice with acute colitis could be masking the effect of
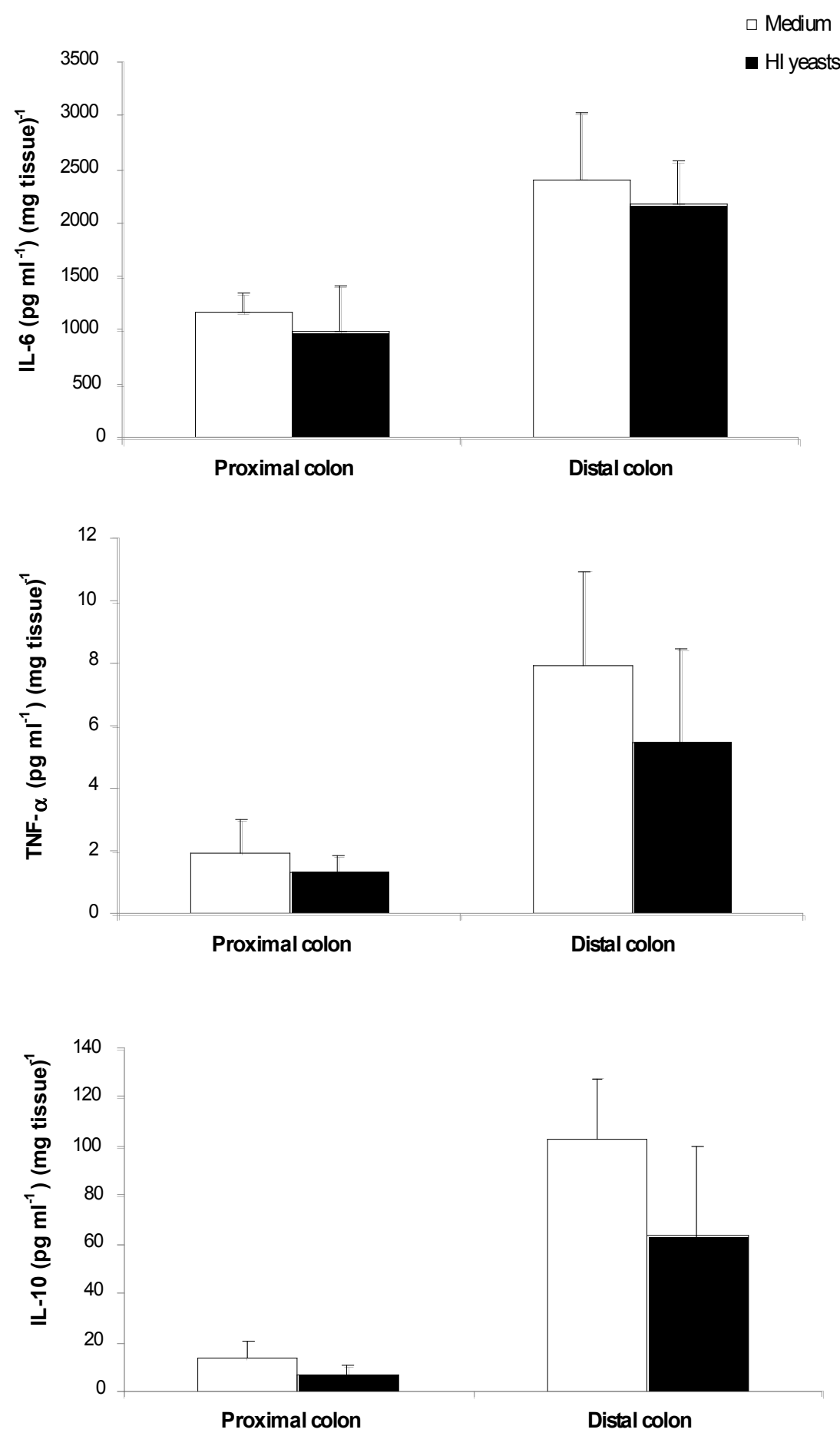

Fig. (4). In vitro colonic production of cytokines by mice with experimental acute colitis. C57BL/6 mice were given $3 \%$ DSS in drinking water for seven days to induce acute experimental colitis. At day 7 mice were sacrificed and sections of $0.5 \mathrm{~cm}$ of proximal and distal colon were obtained and cultured for $24 \mathrm{~h}$ at $37^{\circ} \mathrm{C}$ in RMPI medium or stimulated with $50 \times 10^{6}$ heat-inactivated (HI) S. cerevisiae 10431 yeasts. Levels of IL-6, TNF- $\alpha$ and IL-10 were measured in the supernatants of the cultures by ELISA. The results are shown as mean levels of cytokines [expressed as $\left.\left(\mathrm{pg} \mathrm{ml}^{-1}\right)\left(\mathrm{mg}_{\mathrm{fissue}}\right)^{-1}\right] \pm \mathrm{SD}$. Data are representative of three independent experiments. 
yeasts on the production of cytokines, we performed in vitro assays with colon segments from mice with sublethal colitis.

As shown in Fig. (5A), the addition of heat-inactivated $S$. boulardii yeasts or mannan to the cultures did not produced any significant effect on the in vitro production of the cytokines assayed. The levels of IL- 6 were higher as compared with the levels of TNF- $\alpha$ and IL-10, although were lower than the levels of this cytokine produced by mice with acute colitis (Fig. 4).

We also tested the in vitro production of cytokines in response to inactivated $S$. boulardii yeasts and mannan by colon segments from control healthy mice (Fig. 5B). In these mice the levels of all cytokines were lower as compared with mice with induced colitis, and again the addition of heatinactivated yeasts or mannan did not induce any statistically significant effect on the production of cytokines (Fig. 5B).
Oral Administration of Mannan Increases the Secretion of Cytokines by GI-Tract Cells

To correlate the in vivo and in vitro effects of mannan we assayed the in vitro production of cytokines by colon segments from mice with acute colitis and treated with mannan or PBS (controls). To induce acute colitis mice were given 3\% DSS in drinking water for 7 days and were simultaneously administered with $5 \mathrm{mg}$ of yeast mannan. At day 7 mice were sacrificed and the colon segments were cultured for $24 \mathrm{~h}$ before assessing the in vitro production of IL-6, IL-10 and TNF- $\alpha$.

When compared with PBS-treated mice, colonic segments from mannan-treated mice showed higher levels of cytokines, particularly IL-6 in proximal segments [3-fold increase: from 1100 to $3200 \mathrm{pg} \mathrm{ml}^{-1}$ (mg tissue) ${ }^{-1}$ ] (Fig. 6). TNF- $\alpha$ and IL-10 levels also showed a 2- to 3-fold increase in proximal and distal colon segments, although these levels
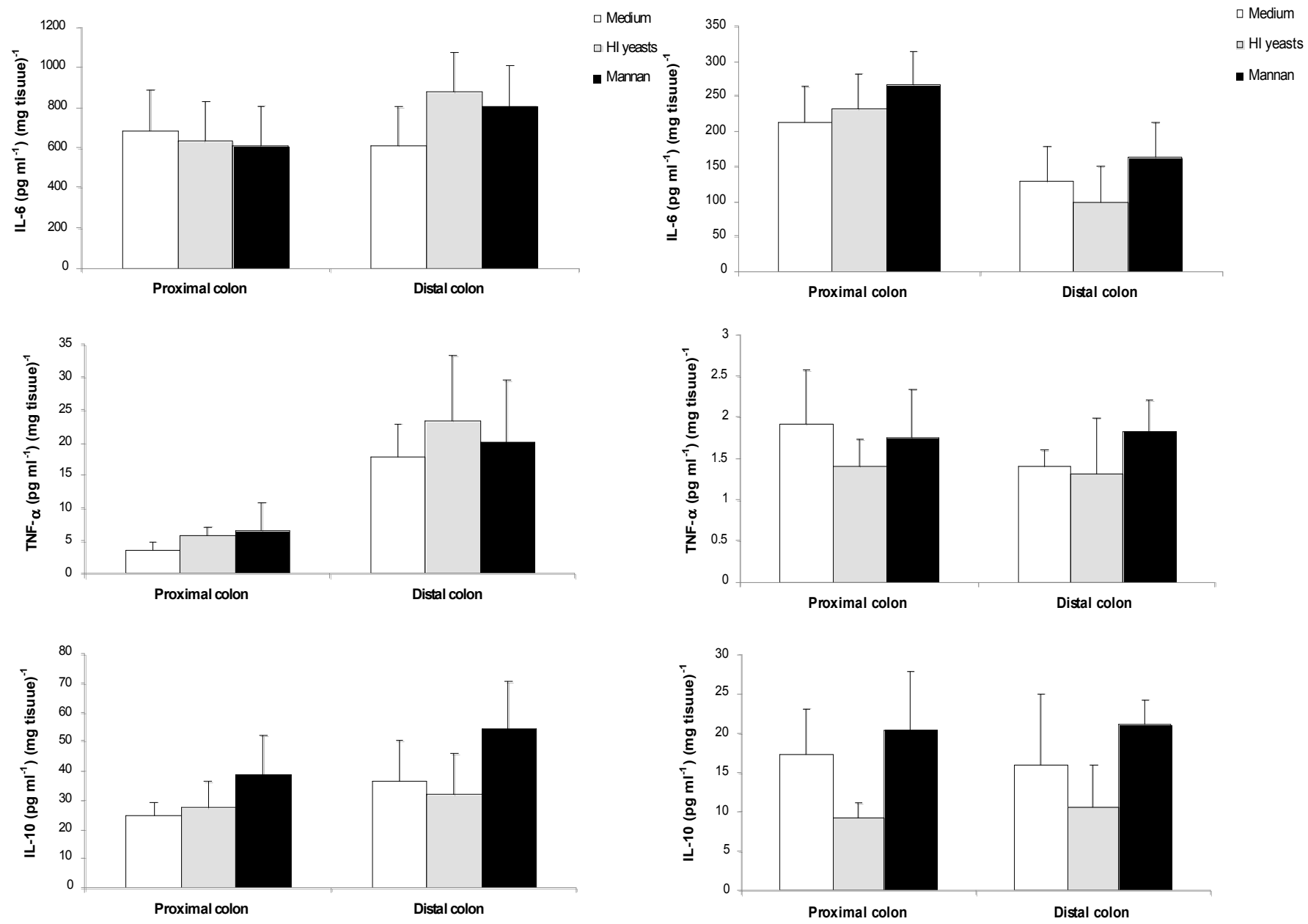

A

B

Fig. (5). In vitro colonic production of cytokines by mice with sublethal colitis in response to inactivated yeasts and mannan. C57BL/6 mice were given $3 \%$ DSS in drinking water until a percentage of loss weight between $7 \%$ and $9 \%$. The mice were then sacrificed and sections of $0.5 \mathrm{~cm}$ of proximal and distal colon were obtained and cultured for $24 \mathrm{~h}$ at $37^{\circ} \mathrm{C}$ in RMPI medium, stimulated with $50 \times 10^{6}$ heatinactivated (HI) $S$. boulardii yeasts or stimulated with $1.5 \mathrm{mg}$ of mannan. (A). The production of cytokines in response to inactivated yeasts or mannan was also measured in segments of proximal and distal colon from healthy mice. (B). Levels of IL-6, TNF- $\alpha$ and IL-10 were measured in the supernatants of the cultures by ELISA. The results are shown as mean levels of cytokines [expressed as $\left(\mathrm{pg} \mathrm{ml}^{-1}\right)\left(\mathrm{mg}^{-1}\right.$ tissue $\left.)^{-1}\right] \pm$ SD. Data are representative of two independent experiments. 


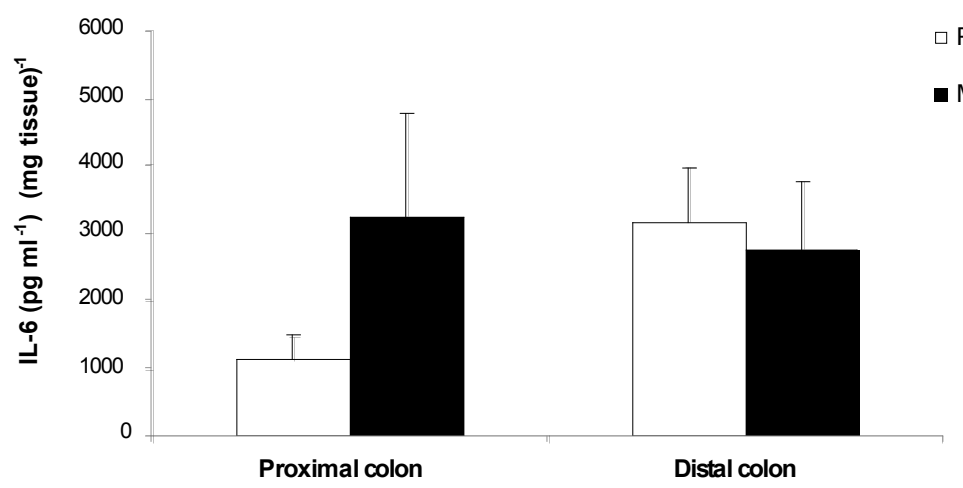

$\square$ PBS-treated mice

Mannantreated mice
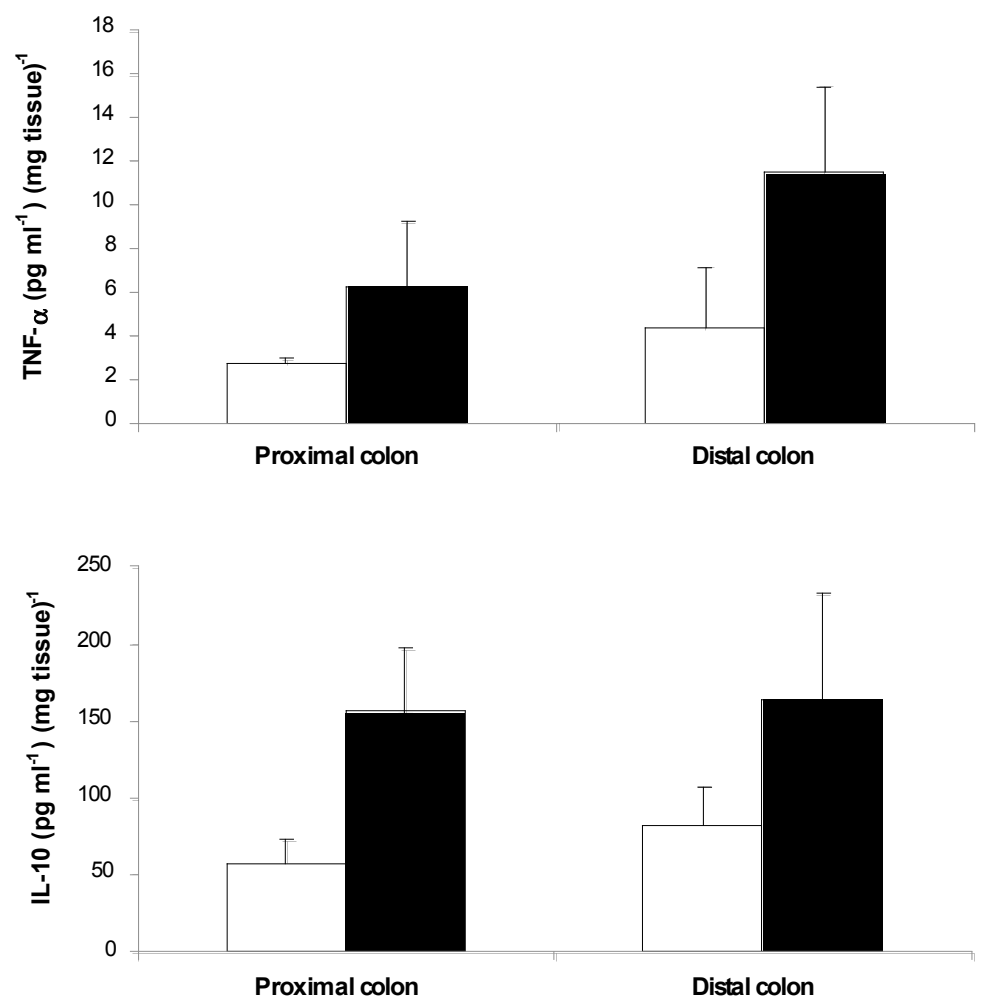

Fig. (6). Effect of the oral administration of mannan on the colonic production of cytokines in mice with experimental acute colitis. C57BL/6 mice were given 3\% DSS in drinking water for seven days to induce acute experimental colitis, and were simultaneously daily administered with $5 \mathrm{mg}$ of yeast mannan in $0.05 \mathrm{ml}$ PBS $(\mathrm{n}=3)$ or with $0.05 \mathrm{ml}$ PBS (control) ( $\mathrm{n}=3$ ). At day 7 mice were sacrificed and sections of $0.5 \mathrm{~cm}$ of proximal and distal colon were obtained and cultured in RMPI medium for $24 \mathrm{~h}$ at $37^{\circ} \mathrm{C}$. Levels of IL-6, TNF- $\alpha$ and IL10 were measured in the supernatants of the cultures by ELISA. The results are shown as mean levels of cytokines [expressed as $\left(\mathrm{pg}^{-1} \mathrm{~m}^{-1}\right)$ $\left.(\mathrm{mg} \text { tissue })^{-1}\right] \pm \mathrm{SD}$. Data are representative of two independent experiments.

were lower than those of IL-6 [3-12 $\mathrm{pg} \mathrm{m}^{-1}$ (mg tissue) $)^{-1}$ for TNF- $\alpha$, and 60-160 $\mathrm{pg} \mathrm{ml}^{-1}$ (mg tissue) ${ }^{-1}$ for IL-10] (Fig. 6). These results suggest that in vivo, mannan treatment does not decrease the secretion of cytokines by GI-tract cells, but rather secretion of cytokines, particularly IL-6, is actually enhanced, probably depending on the degree of tissue injury. However, due to the high variability of cytokine levels obtained between samples from individual mice, the statistical significance of these differences should be further assessed.

\section{DISCUSSION}

The incidence of Crohn's disease and ulcerative colitis has increased greatly in recent years, converting these IBDs in a major public health problem [29]. The host microbial environment plays an important role in the development of IBD, so the administration of probiotics to manipulate the intestinal microbiota and reduce the inflammatory response constitutes one potential option for therapeutic intervention $[2,7]$. As the fungal species $S$. boulardii, used as commercial probiotic, is recognized to have biotherapeutic effects on intestinal mucosa $[21,22]$ and it is well established that TLRs, receptors involved in controlling the intestinal epithelial homeostasis [10], recognize microbial-associated molecular paterns from fungi $[18,19]$, in this work we have studied the effect of oral administration of fungal ligands on the amelioration of colitis using a murine model of DSSinduced colitis. It should be noted in this context that mimetics of bacterial DNA, given orally or subcutaneously, 
protect mice from experimental colitis via a TLR9-dependent mechanism $[12,13]$.

Protection assays on mice with an experimentally DSSinduced acute colitis showed that the oral administration of fungal ligands, either $S$. cerevisiae 10431 cells, S. boulardii cells or soluble yeast cell wall-derived mannan, have no effect on the protection from mortality caused by the chemically-induced intestinal injury, even if the treatment of mice starts during the induction of colitis. These results do not support the hypothesis that yeast cells contribute to the in vivo protection of the GI tract during inflammatory diseases. Previously, Dalmasso and colleagues had shown that $S$. boulardii has a beneficial effect in the treatment of IBS [30]. Using a chronic IBD model on immunodeficient mice they showed that the oral treatment with lyophilized $S$. boulardii limits the infiltration of Th1 cells in the inflamed colon and the amplification of inflammation induced by proinflammatory cytokine production. Moreover, using a model of trinitrobenzene sulfonic acid-induced colitis in rats, other authors had shown that $S$. boulardii is able to ameliorate in vivo the symptoms of the disease [31]. The high degree of intestinal injury developed in our model of acute colitis, proved by the high loss of weight and by the low survival rate, could partially explain that the treatment with viable yeast cells showed any beneficial effect on the amelioration of the disease. However, when we performed similar assays in a model of sublethal colitis, in which the mice present a higher survival rate after the DSS treatment, we also did not find any biotherapeutic effect of the oral administration of viable $S$. boulardii yeasts.

The epithelial damage of the GI tract was evidenced by an augment in the colonic production of cytokines. Cytokines play a central role in the modulation of the intestinal immune system. In IBD it has been described a disturbed balance between proinflammatory and antiinflammatory cytokines. In both $\mathrm{UC}$ and $\mathrm{CD}$, a polarized immune Th1 response (marked by upregulation of IL-6, TNF- $\alpha$, IL-1 $\beta$ and IFN- $\gamma$ ) and Th17 response (marked by IL17 secretion) was reported, whereas patients with UC exhibit an added contribution of $\mathrm{Th} 2$ responses (characterized by secretion of IL-4, IL-5, and IL-13) [32, 33]. In addition, IFN$\gamma$ is causatively involved in experimental inflammatory bowel disease in mice [34], and TLRs play a significant role in the modulation of IFN $-\gamma$ production in response to fungal ligands [19,35].

There is growing evidence that an IL-6 plays a crucial role in the uncontrolled intestinal inflammatory process of IBD [33, 36-39]. In our experimental model the production of IL- 6 by proximal colon fragments from mice with DSSinduced sublethal colitis present a 3-fold increase compared with healthy mice, whereas this augment reached a 5-fold increase in distal colon fragments. Moreover, mice with acute colitis present higher levels of IL-6 when compare with healthy mice and mice with sublethal colitis; the colonic production of the cytokine in mice with acute colitis was about 5-fold increased in proximal colon segments and 19 -fold in distal colon relative to healthy mice. The addition of mannan or heat-inactivated yeast cells, either S. cerevisiae or $S$. boulardii, to the culture did not produce any significant effect on the in vitro production of IL- 6 by colon segments from healthy mice or mice with induced colitis. This correlates with the fact that oral administration of fungal ligands to mice with colitis did not contribute to the amelioration of the symptoms of the disease or to the protection from mortality. The non-therapeutic effect of fungal ligands was also confirmed by the observation that mice with acute induced-colitis and treated simultaneously with yeast mannan present an increase in the colonic levels of IL-6 when compared with control mice treated with PBS.

TNF- $\alpha$ is an important mediator of inflammation and, although different results have been described for the mucosal levels of TNF- $\alpha$ during IBD, specific anti-TNF- $\alpha$ agents have been widely used in the treatment of IBD in the last decade $[32,40]$, whereas the importance of IL-10 in IBD was former demonstrated using IL-10-deficient mice, as these mice develop chronic enterocolitis that can be prevented by administration of IL-10 [41]. In our experimental conditions, the levels of the proinflammatory cytokine TNF$\alpha$ and the anti-inflammatory cytokine IL-10 were lower when compared with the production of IL- 6 , but we also found that fungal ligands had no significant effect on the production of these cytokines.

\section{CONCLUSION}

Our results show that, in the experimental conditions assayed, fungal ligands do not have a biotherapeutic effect on the protection of mice from disease in a model of chemically-induced colitis in immunocompetent mice. The experimental model of colitis used in this work causes a high degree of intestinal injury, proved by low survival rates and high level of proinflammatory cytokines, which can partially explain the inability of fungal ligands to ameliorate the disease. Moreover, the potential protective effect of the fungal ligands may be masked by the presence of commensal bacteria and other factors acting during the in vivo process that may result in mannan and yeasts degradation. Further assays using (i) mice depleted of commensal bacteria as a consequence of long-term treatment with antibacterial antibiotics, and/or (ii) enteric coating of mannan/yeast to prevent release before it reaches the intestine should be performed prior to rule out the potential protective effect of yeast ligands during experimentally induced colitis. Alternatively, systemic administration of fungal ligands for TLRs may be also considered, as activation of TLR3 on cells that are accessible by systemic, but not oral, administration of synthetic viral RNA, a TLR3 ligand, causes protection against the acute inflammation upon damage of the gut epithelium in a mouse model of DSS-induced colitis [14].

\section{ACKNOWLEDGEMENTS}

This work was supported by grant PI080556 (Instituto de Salud Carlos III; Ministerio de Ciencia e Innovación, Spain). Celia Murciano is supported by a Federetion of Europeon Microbiological Societies (FEMS) Advanced Fellowship. Alberto Yáñez was recipient of a fellowship from the Ministerio de Educación y Ciencia. We are grateful to the SCSIE (Servicio Central de Soporte a la Investigación Experimental) of the University of Valencia, for technical assistance. 


\section{CONFLICT OF INTEREST}

None declared.

\section{REFERENCES}

[1] Danese S, Fiocchi C. Etiopathogenesis of inflammatory bowel diseases. World J Gastroenterol 2006; 12(30): 4807-12.

[2] Geier MS, Butler RN, Howarth GS. Inflammatory bowel disease: current insights into pathogenesis and new therapeutic options; probiotics, prebiotics and synbiotics. Int J Food Microbiol 2007; 115(1): 1-11.

[3] Chichlowski M, Hale LP. Bacterial-mucosal interactions in inflammatory bowel disease: an alliance gone bad. Am J Physiol Gastrointest Liver Physiol 2008; 295(6): 1139-49.

[4] Okayasu I, Hatakeyama S, Yamada M, Ohkusa T, Inagaki Y, Nakaya R. A novel method in the induction of reliable experimental acute and chronic ulcerative colitis in mice. Gastroenterology 1990; 98(3): 694-702.

[5] Melgar S, Karlsson A, Michaelsson E. Acute colitis induced by dextran sulfate sodium progresses to chronicity in C57BL/6 but not in $\mathrm{BALB} / \mathrm{c}$ mice: correlation between symptoms and inflammation. Am J Physiol Gastrointest Liver Physiol 2005; 288(6): 1328-38.

[6] Fuller R. Probiotics in man and animals. J Appl Bacteriol 1989; 66(5): 365-78

[7] Ewaschuk JB, Dieleman LA. Probiotics and prebiotics in chronic inflammatory bowel diseases. World J Gastroenterol 2006; 12(37): 5941-50.

[8] Takeda K, Akira S. Toll-like receptors in innate immunity. Int Immunol 2005; 17(1): 1-14

[9] O'Neill LA. The interleukin-1 receptor/Toll-like receptor superfamily: 10 years of progress. Immunol Rev 2008; 226: 10-8.

[10] Rakoff-Nahoum S, Paglino J, Eslami-Varzaneh F, Edberg S, Medzhitov R. Recognition of commensal microflora by toll-like receptors is required for intestinal homeostasis. Cell 2004; 118(2): $229-41$.

[11] Rumbo M, Nempont C, Kraehenbuhl JP, Sirard JC. Mucosal interplay among commensal and pathogenic bacteria: lessons from flagellin and Toll-like receptor 5. FEBS Lett 2006; 580(12): 297684

[12] Katakura K, Lee J, Rachmilewitz D, Li G, Eckmann L, Raz E. Toll-like receptor 9-induced type I IFN protects mice from experimental colitis. J Clin Invest 2005; 115(3): 695-702.

[13] Lee J, Rachmilewitz D, Raz E. Homeostatic effects of TLR9 signaling in experimental colitis. Ann N Y Acad Sci 2006; 1072: $351-5$.

[14] Vijay-Kumar M, Wu H, Aitken J, et al. Activation of toll-like receptor 3 protects against DSS-induced acute colitis. Inflamm Bowel Dis 2007; 13(7): 856-64

[15] Brandl K, Sun L, Neppl C, et al. MyD88 signaling in nonhematopoietic cells protects mice against induced colitis by regulating specific EGF receptor ligands. Proc Natl Acad Sci USA 2010; 107(46): 19967-72.

[16] O'Neill LA. A feed-forward loop involving hyaluronic acid and toll-like receptor-4 as a treatment for colitis? Gastroenterology 2009; 137(6): 1889-91.

[17] Zheng L, Riehl TE, Stenson WF. Regulation of colonic epithelial repair in mice by Toll-like receptors and hyaluronic acid. Gastroenterology 2009; 137(6): 2041-51.

[18] Netea MG, Brown GD, Kullberg BJ, Gow NA. An integrated model of the recognition of Candida albicans by the innate immune system. Nat Rev Microbiol 2008; 6(1): 67-78.

[19] Gil ML, Gozalbo D. Role of Toll-like receptors in systemic Candida albicans infections. Front Biosci 2009; 14: 570-82.

[20] Gozalbo D, Roig P, Villamon E, Gil ML. Candida and candidiasis: the cell wall as a potential molecular target for antifungal therapy. Curr Drug Targets Infect Disord 2004; 4(2): 117-35.
Buts JP, De Keyser N. Effects of Saccharomyces boulardii on intestinal mucosa. Dig Dis Sci 2006; 51(8): 1485-92.

Doron SI, Hibberd PL, Gorbach SL. Probiotics for prevention of antibiotic-associated diarrhea. J Clin Gastroenterol 2008; 42 (Suppl 2): S58-63.

[23] Jawhara S, Poulain D. Saccharomyces boulardii decreases inflammation and intestinal colonization by Candida albicans in a mouse model of chemically-induced colitis. Med Mycol 2007; 45(8): 691-700.

[24] Main J, McKenzie H, Yeaman GR, et al. Antibody to Saccharomyces cerevisiae (bakers' yeast) in Crohn's disease. BMJ 1988; 297(6656): 1105-6.

[25] Standaert-Vitse A, Jouault T, Vandewalle P, et al. Candida albicans is an immunogen for anti-Saccharomyces cerevisiae antibody markers of Crohn's disease. Gastroenterology 2006; 130(6): 1764-75.

[26] Yanez A, Murciano C, Llopis S, et al. In vivo and in vitro studies on virulence and host responses to Saccharomyces cerevisiae clinical and non-clinical isolates. Open Mycol J 2009; 3: 37-47.

[27] Villamon E, Gozalbo D, Roig P, O'Connor JE, Fradelizi D, Gil ML. Toll-like receptor-2 is essential in murine defenses against Candida albicans infections. Microbes Infect 2004; 6(1): 1-7.

[28] Murciano C, Yanez A, Gil ML, Gozalbo D. Both viable and killed Candida albicans cells induce in vitro production of TNF-alpha and IFN-gamma in murine cells through a TLR2-dependent signalling. Eur Cytokine Netw 2007; 18(1): 38-43.

[29] Gismera CS, Aladren BS. Inflammatory bowel diseases: a disease(s) of modern times? Is incidence still increasing? World J Gastroenterol 2008; 14(36): 5491-8.

[30] Dalmasso G, Cottrez F, Imbert V, et al. Saccharomyces boulardi inhibits inflammatory bowel disease by trapping $\mathrm{T}$ cells in mesenteric lymph nodes. Gastroenterology 2006; 131(6): 1812-25.

[31] Lee SK, Kim YW, Chi SG, Joo YS, Kim HJ. The effect of Saccharomyces boulardii on human colon cells and inflammation in rats with trinitrobenzene sulfonic acid-induced colitis. Dig Dis Sci 2009; 54(2): 255-63.

[32] Dharmani P, Chadee K. Biologic therapies against inflammatory bowel disease: a dysregulated immune system and the cross talk with gastrointestinal mucosa hold the key. Curr Mol Pharmacol 2008; 1(3): 195-212.

[33] Rogler G, Andus T. Cytokines in inflammatory bowel disease. World J Surg 1998; 22(4): 382-9.

[34] Ito R, Shin-Ya M, Kishida T, et al. Interferon-gamma is causatively involved in experimental inflammatory bowel disease in mice. Clin Exp Immunol 2006; 146(2): 330-8.

[35] Gozalbo D, Gil ML. IFN-gamma in Candida albicans infections. Front Biosci 2009; 14: 1970-8.

[36] Ito H, Takazoe M, Fukuda Y, et al. A pilot randomized trial of a human anti-interleukin-6 receptor monoclonal antibody in active Crohn's disease. Gastroenterology 2004; 126(4): 989-96; discussion 947.

[37] Kusugami K, Fukatsu A, Tanimoto M, et al. Elevation of interleukin-6 in inflammatory bowel disease is macrophage- and epithelial cell-dependent. Dig Dis Sci 1995; 40(5): 949-59.

[38] Mitsuyama K, Sasaki E, Toyonaga A, et al. Colonic mucosal interleukin-6 in inflammatory bowel disease. Digestion 1991, 50(2): 104-11.

[39] Mitsuyama K, Sata M, Rose-John S. Interleukin-6 trans-signaling in inflammatory bowel disease. Cytokine Growth Factor Rev 2006; 17(6): 451-61.

[40] Liu Z, Jiu J, Liu S, Fa X, Li F, Du Y. Blockage of tumor necrosis factor prevents intestinal mucosal inflammation through downregulation of interleukin-23 secretion. J Autoimmun 2007; 29(2-3): 187-94.

[41] Kuhn R, Lohler J, Rennick D, Rajewsky K, Muller W. Interleukin10-deficient mice develop chronic enterocolitis. Cell 1993; 75(2): 263-74. 